\title{
Napoli, Biblioteca Nazionale Vittorio Emanuele III, III D 37
}

Costantinopoli, XIV sec.

ff. I-III, 1-286, IV-VI; ff. di guardia cartacei: sul f. IIr vi è il pinax con il contenuto del codice; sul f. $1 \mathrm{r}$ indicazione a penna: as $n^{\circ} 15$ Logica porfirii et Aristotelis organum totum cum scolys; num. recente al centro del marg. inferiore a matita; cart.; fil. ff. $4,28,33,34,35,37,79,81,83,85,90,92,93,170,171,172,173$ simile a fruit $(\mathrm{M} / \mathrm{Tr} \mathrm{nr}$. 4204, 1369), ff. 5, 8, 10, 11 simile a lettre (M/Tr nr. 5652, 1361), ff. 14, 15, 19, 22, 23, 24, 26, 39 simile chapeau (M/Tr nr. 2386, 1360), f. 17 simile a cercles (M/Tr nr. 2075, 1364), ff. 45, 46, 47, 48, 50, 54, 56, 58, 60, 62, 64, 66, 248 simile a $\operatorname{arc}(\mathrm{M} / \mathrm{Tr}$ nrr. 347-356, 1363), ff. 74, 75, 76, 77, 88, 94, 95, 96, 98, 105, 107, 108, 109, 113, 115, 116, 117 simile a fleur (M/Tr nr. 4095, 1376), ff. 121, 124, 125, 127, 128, 129, 133, 135, 136, 138, 141, 142, 143, 144, 146, 153, 155, 156, 157, 158, 161, 163, 164 simile a cerf (M/Tr nr. 2245, 1370), ff. 174, 178, 179, 180, 185, 187, 189, 192, 193, 197, 198, 206, 207, 209, 211, 214, 215 , 216, 218, 223, 226, 227, 229, 231, 232, 234, 237, 239, 242, 243, 245, 269, 270, 271, 272, 278 , 279, 280, 283, 285, 286 simile a ange (M/Tr nr. 190, 1371), ff. 200, 201 simile a griffon (M/Tr nr. 4547, 1365/1370), f. 204 simile a huchet (M/Tr nr. 4884, XIV), ff. 249, 250, 252, 254, 258, 259, 260, 264 simile a casque (M/Tr nr. 1786, 1360-1375); $1^{4}(4), 2^{8}(12)$, $3^{10}(22) 4^{8+1}(31), 5^{8}(39), 6^{2+4}(45), 7-31^{8}(245), 32^{4-1}(248), 33-36^{8}(280), 37^{6}(286)$; num. fasc. visibile nell'angolo del marg. sup. est. del recto del primo e nel marg. inf. int. del verso dell'ultimo foglio di alcuni fascicoli, numerati da $\beta^{\prime}$ a $\lambda \varsigma^{\prime}$; ff. $5 \mathrm{r}-15 \mathrm{v}, 17 \mathrm{r}$ 26v, 28r-36r, 38r-50r, 52r, 53rv, 54v-56r, 57r-172v, 173v, 174v-248r, 249v-285r: mm 295 × $223=20$ // 250 // $25 \times 18$ // 10 // 119 // 10 // 47 // 23, a piena pagina, 1l. 25-46, unità di rigatura mm 3-5, rigatura visibile sul recto di alcuni fogli tracciata a secco (f. 69); ff. 1r-4v, 16rv, 27rv, 36v-37v, 50v-51v, 52v, 54r, 56v, 173r, 249r: mm $295 \times 223=23 / / 241$ // $31 \times 19$ // 170 // 33, a piena pagina, ll. 53, unità di rigatura mm 3, rigatura visibile sul recto di alcuni fogli. Sono bianchi i fogli 248v, 285v-286v. Legatura farnesiana; sul dorso sono presenti i gigli e il titolo in oro.

\section{Scrittura}

Hanno collaborato alla trascrizione del codice due copisti: <Isacco Argiro (Bianconi 2008, 359-360)> (testo principale dei ff. 1r-4v, 29v-37v, 43v-248v, 1. 2, 249r-285r, 1. 13); Anonimo A (ff. 5r-26v, 28r-29r, 38r-43r - solo questi ultimi fogli segnalati anche in Formentin 2015, 168 - e la maggior parte degli scoli presenti nei ff. 5r-23v). 


\section{Decorazione}

Rubricatio, alcuni scoli inseriti nel testo, schemi in inchiostro rosso (passim).

\section{Interventi successivi}

Interventi di mani posteriori ai fogli $17 \mathrm{r}$ e $32 \mathrm{v}$. Al f. 285r, dopo la sottoscrizione, una mano del XV secolo ha scritto una nota di possesso, ora depennata e dunque poco

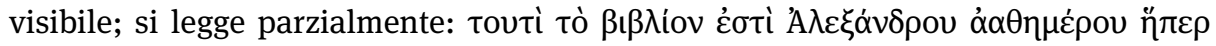

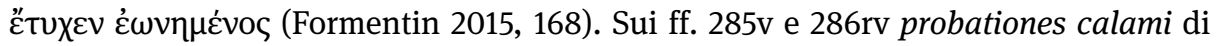
una mano del XVI secolo; al f. 286r, una mano ancora più recente ha scritto in latino dieci righe De demostratione et syllogismo.

\section{Contenuto}

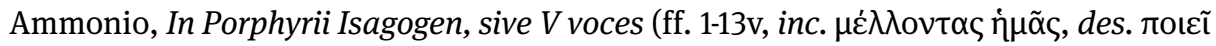

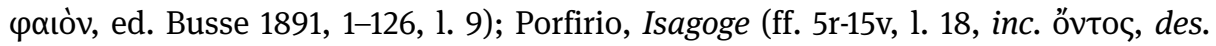

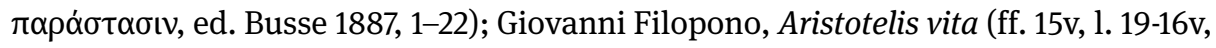

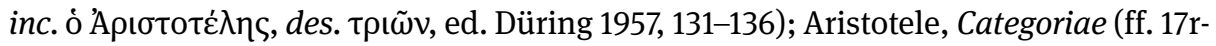
38r, 1. 3, inc. ò $\mu \omega \dot{v v \nu \mu \alpha ~ \lambda \varepsilon ́ \gamma \varepsilon \tau \alpha ı, ~ d e s . ~ \alpha ̆ \pi \alpha v \tau \varepsilon \varsigma ~ к \alpha \tau \eta \rho i \theta \mu \eta \nu \tau \alpha ı, ~ e d . ~ M i n i o ~ P a l u e l l o ~ 1961, ~}$ 3-45); excerpta ex Giovanni Filopono, In Aristotelis categorias commentarium (f. 15r,

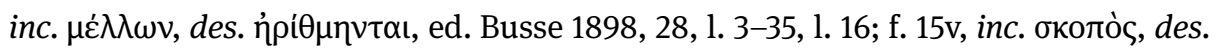

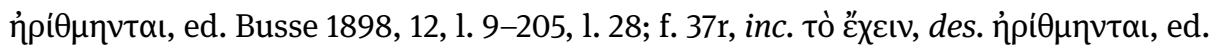
Busse 1989, 204, 1. 24-205, 1. 28); Aristotele, De interpretatione (ff. 38r-50r, 1. 19, inc.

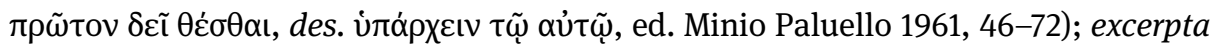
ex Ammonio, In Aristotelis librum de interpretatione commentarium (ff. 37v-51r, inc.

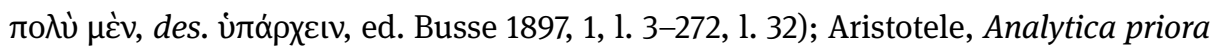

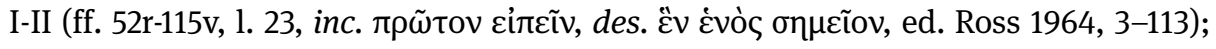
excerpta ex Giovanni Filopono, In Aristotelis analytica priora commentaria (ff. 51v-115v,

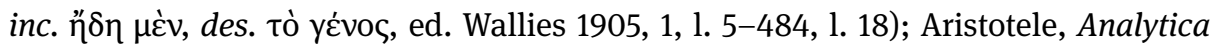

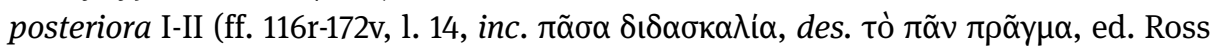
1964, 114-183); Giovanni Filopono, In Aristotelis analytica posteriora commenta-

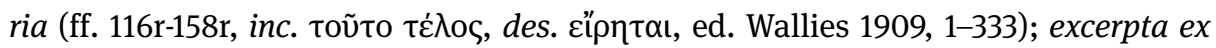
Anonimo, In Aristotelis analytica posteriora commentaria (ff. 158r-172v, inc. $\delta \mathrm{i} \alpha \lambda \alpha \beta \dot{\omega} v$,

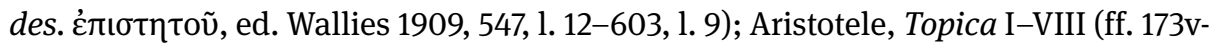

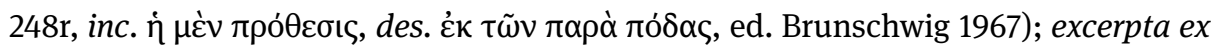
Alessandro di Afrodisia, In Aristotelis topicorum libros octo commentaria (ff. 173r-248v,

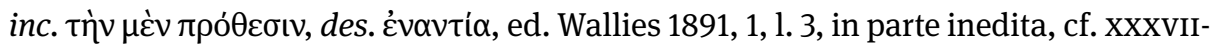

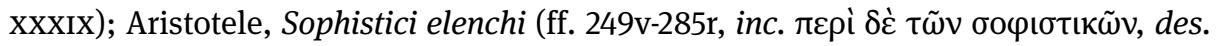

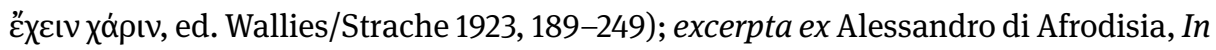




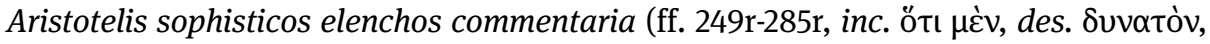
ed. Wallies 1898, 189, 1. 16-198, 1. 15).

\section{Relazioni stemmatiche}

Per quanto concerne la Vita di Aristotele, trasmessa nel complesso da 31 manoscritti, il Neapolitanus è considerato il migliore testimone della famiglia E, formata da 6 manoscritti e caratterizzata dalla particolarità di far precedere la Vita da una breve prefazione alle Categorie (Düring 1957, 126).

\section{Storia del codice}

Il codice, appartenuto alla biblioteca farnesiana, è il nr. 6 nell'antico inventario stilato il 10 gennaio del 1567 (Pernot 1979, 494-495), in occasione della presa in custodia della biblioteca da parte di Fulvio Orsini. Tra il 1641 e il 1653 il codice, insieme a tutta la biblioteca, fu trasferito a Parma, dove fu munito, in accordo con la campagna di rilegatura che interessò l'intera collezione, dell'attuale legatura in vitello bruno, con gigli dorati sul dorso (Guerrieri 1962, xIx). Fu probabilmente vergata in quell'occasione la nota al f. 1r con l'indicazione del numero e del contenuto (Formentin 2008, 79 n. 3). La collezione rimase nel ducato di Parma fino al 1734, quando Carlo III di Borbone portò con sé a Napoli la biblioteca ereditata dalla madre (Formentin 2008, 79 n. 3).

\section{Bibliografia}

\section{Cataloghi}

Baffi (1796) 782, n. 129; Cirillo (1832) II, 428-430 n. 321; Formentin (2015) 167-169.

\section{Edizioni}

Busse (1887); Busse (1891); Busse (1897); Busse (1898); Brunschwig (1967); Düring (1957); Minio Paluello (1961); Ross (1964); Wallies (1891); Wallies (1898); Wallies (1905); Wallies (1909); Wallies/Strache (1923). 


\section{Codicologia e paleografia}

Bianconi (2008) 359-360; Brunschwig (1967) I, cxv n. 1; Formentin (2008) 80; Guerrieri (1962) XIX; Pernot (1979) 494-495, 501; Wartelle (1963) 86, n. 1187.

\section{Riproduzioni}

Bianconi (2008) tavv. 12-14; Formentin (2015) tav. XII. 